\title{
ADJUNCTION OF ROOTS TO NILPOTENT GROUPS
}

\author{
by R. B. J. T. ALLENBY
}

(Received 3 September, 1964)

1. Introduction. For any nilpotent group $B$ of class $c$ and any given element $h$ of $B$ generating the subgroup $H$, Wiegold [1] has shown that if, in addition, $[B, H]$ has exponent $p^{r}$ for some prime $p$ and integer $r$, then $B$ can be embedded in a nilpotent group $G$ such that $G$ also contains a $p^{s}$ th root for $h(s \geqq 1)$. In fact, Wiegold has gone further and calculated an upper bound for the class of $G$ in terms of the variables $c, p, r, s$.

In a thesis presented to the University of Manchester for the degree of Master of Technical Science, I was able to improve on Wiegold's bound (which is a cubic in the variables $c, p^{s}, r$ ) in the case in which $B$ is metabelian, obtaining a bound for the class of $G$ which is a quadratic in the variables and so is much smaller for large values of the variables. Here $I$ have been able to extend this result, using different (and very much simpler) commutator calculations, to a wider class of groups (see Theorem 3.3.1), and by the same method to reduce the bound in the most general case to about one third of the original value for large values of the variables (Theorem 4.1).

In $\S 5$ we deal with the problem of lower bounds for the nilpotency classes of the embedding groups under consideration. In [1], Wiegold constructed, for each odd integer $r \geqq 3$, a class 2 group $B_{r}$, containing an element $h$ and having the property that $[B, H]$ has exponent $p^{r}$, such that no nilpotent group $G_{r}$ embedding $B_{r}$ and a $p$ th root for $h$ can have class less than $\frac{1}{2}(r+5)$. If we consider only those groups $G_{r}$ which embed $B_{r}$, contain a $p$ th root for $h$, and have the same solubility length as $B_{r}$ (this is not an unnatural thing to ask; see $\S 3$ ), then we can further show that the groups $G_{r}$ must have class at least $p+1$.

$\S 2$ deals with preliminaries and also contains a few easy results required later.

Finally, I should like to express my gratitude to my supervisor Dr James Wiegold for the assistance he has willingly given me in preparing both my thesis and this paper.

I am indebted to the referee for his valuable comments.

\section{Notation and preliminaries.}

2.1. The notation will be that usually used in group-theoretical writings. For example, we use the usual left-norming for commutators, so that for arbitrary elements $x_{1}, x_{2}, \ldots, x_{n}$, $x_{n+1}$ of a group $G$,

and inductively

$$
\left[x_{1}, x_{2}\right]=x_{1}^{-1} x_{2}^{-1} x_{1} x_{2}
$$

$$
\left[x_{1}, x_{2}, \ldots, x_{n}, x_{n+1}\right]=\left[\left[x_{1}, x_{2}, \ldots, x_{n}\right], x_{n+1}\right] \text {. }
$$

Similarly, for subsets $X_{1}, X_{2}, \ldots, X_{n}, X_{n+1}$ of $G,\left[X_{1}, X_{2}\right]$ is the subgroup of $G$ generated by all commutators $\left[x_{1}, x_{2}\right]$ with $x_{1} \in X_{1}, x_{2} \in X_{2}$, and inductively

$$
\left[X_{1}, X_{2}, \ldots, X_{n}, X_{n+1}\right]=\left[\left[X_{1}, X_{2}, \ldots, X_{n}\right], X_{n+1}\right] \text {. }
$$


If $x_{1}=x, x_{2}=x_{3}=\ldots=x_{n+1}=y$ we shall write $\left[x_{1}, x_{2}, \ldots, x_{n}, x_{n+1}\right]$ as $[x, n y]$, and similarly for subsets.

Two well-known commutator identities we require are

$$
\begin{aligned}
& {[x, y z]=[x, z][x, y]^{z},} \\
& {[x y, z]=[x, z][x, z, y][y, z]}
\end{aligned}
$$

for all $x, y, z$ in a group $G$.

The lower and upper central series of a group $G$ will be denoted respectively by

$$
\begin{aligned}
& G=\gamma_{1}(G) \supseteq \gamma_{2}(G) \supseteq \ldots \supseteq \gamma_{i}(G) \supseteq \ldots, \\
& E=\zeta_{0}(G) \subseteq \zeta_{1}(G) \subseteq \ldots \subseteq \zeta_{i}(G) \subseteq \ldots,
\end{aligned}
$$

where $E$ is the unit subgroup of $G$.

Throughout $B$ will denote a nilpotent group, $c$ its class, and $h$ the element of $B$ to which we adjoin roots. $H$ will be the subgroup of $B$ generated by $h$.

2.2. Let $U, V$ be groups with $V$ cyclic of order $q$ ( $q$ arbitrary) generated by the element $\alpha$. For each $i=0,1, \ldots, q-1$, let $U_{i}$ be an isomorphic copy of $U$ and let the element of $U_{i}$ corresponding to the element $u$ of $U$ be denoted by $u_{i}$. Writing $\widetilde{U}$ for the direct product of the $U_{i}$, we define the wreath product of $U$ by $V$ to be the group $W=U \mathrm{wr} V$ given by

$$
W=\operatorname{gp}\left\{\bar{U}, V ; \alpha^{-j} u_{i} \alpha^{j}=u_{i+j}, u \in U, 0 \leqq j<q, i+j \text { reduced } \bmod q\right\} .
$$

It follows easily that every element of $W$ can be written in the form

$$
w=u(0)_{0} u(1)_{1} \ldots u(q-1)_{q-1} \alpha^{i},
$$

where $u(0), u(1), \ldots, u(q-1)$ are elements of $U$ and $0 \leqq i<q$. The set of all such elements for which $u(0)=u(1)=\ldots=u(q-1)$ and $i=0$ forms a subgroup $U^{0}$ of $W$ called the diagonal of $W$. Clearly $U^{0} \cong U$. If $u^{0}$ is any element of $U^{0}$, it is easy to show that $\alpha^{-1} u^{0} \alpha=u^{0}$; thus every power of $\alpha$ commutes with every element of $U^{0}$. If $u \in U$, it follows from Lemma 2.2.3 (below) that $\left(u_{0} \alpha\right)^{q}=u_{0} u_{1} \ldots u_{q-1}$ so that, on identifying $U$ and $U^{0}$, the element $u_{0} \alpha$ is a $q$ th root for $u$ in $W$. (This method of root adjunction is due to Baumslag [2].)

Finally, we note that with this notation $\left[x_{i}, y_{i}\right]=[x, y]_{i}$ for all $x, y \in U, 0 \leqq i<q$.

The following lemma is easily proved by induction on $t$.

LEMmA 2.2.1. If $W=U \operatorname{wr} V\left(V=\operatorname{gp}\left\{\alpha: \alpha^{q}=1\right\}\right)$, then, for any $u \in U$ and any arbitrary positive integer $t$,

$$
\left[u_{0}, t \alpha\right]=u_{0}^{(-1)^{t}} u_{1}^{(-1)^{t+1}(t: 1)} \ldots u_{i}^{(-1)^{t+1}(t: i)} \ldots u_{t},
$$

where $(t: i)$ denotes the binomial coefficient $\left(\begin{array}{l}t \\ i\end{array}\right)$. (Because of the frequent occurrence of binomial coefficients in exponents, we shall use this notation throughout the present paper.)

This is Lemma 3.1 of Liebeck [3], where it is apparently stated incorrectly; there is a slight mistake in the signs of the indices. 
If $t \geqq q$ we can simplify $\left[u_{0}, t \alpha\right]$ by observing that $U_{i}, U_{i+q}, U_{i+2 q}, \ldots$ are all the same subgroup $U_{i}$ of $W$. It follows that we may write $\left[u_{0}, t \alpha\right]$ as

$$
\left[u_{0}, t \alpha\right]=u_{0}^{\lambda(0, t)} u_{1}^{\lambda(1, t)} \ldots u_{q-1}^{\lambda(q-1, t)}=\prod_{i=0}^{q-1} u_{i}^{\lambda(i, t)},
$$

where

$$
\lambda(i, t)=(-1)^{t+i}(t: i)+(-1)^{t+i+q}(t: i+q)+\ldots \quad(t \geqq i, 0 \leqq i<q) .
$$

Now using induction on $r$ we can prove

LEMMA 2.2.3. For any non-negative integer $r$ such that $r=(k-1) q+i(k \geqq 1,0 \leqq i<q)$ and any element $u$ of $U$, we have

$$
\left(u_{0} \alpha\right)^{r}=\alpha^{r} . u_{1}^{k} u_{2}^{k} \ldots u_{i}^{k} u_{i+1}^{k-1} \ldots u_{q-1}^{k-1} u_{0}^{k-1} .
$$

Note that the components $u_{i}, u_{j}$ are permutable for $i \neq j$ since they belong to the direct product $\bar{U}$.

Finally in this section we establish

LemMa 2.2.4. Let $G$ be a group and $S$ any subgroup of $G$. Then if $g, x$ are arbitrary elements of $G,[G, S]$, respectively, we have, for $n>0$,

$\left[x^{n}, g\right] \equiv[x, g]^{n}[x, g, x]^{(n: 2)} \ldots[x, g,(n-1) x] \bmod [G,[G, S],[G, S],[G, S, G]]$.

Proof. We note that all elements occurring on the right-hand side belong to normal subgroups (namely $[[G, S], G],[[G, S], G,[G, S]], \ldots$ ) of $G$. (For the normality of $[G, S]$ in $G$, see Golovin [4]). The proof proceeds by induction; we assume the result for $n=k$, then write

$$
\left[x^{k+1}, g\right]=\left[x^{k}, g\right]\left[x^{k}, g, x\right][x, g]
$$

and use (2.1.2). The subgroup $[G,[G, S],[G, S],[G, S, G]]$ is chosen simply because it is the smallest (sensible) subgroup of $G$ modulo which all the elements on the right-hand side of (2.2.5) commute.

Since $[x, g, l x] \in[G,[G, S],[G, S],[G, S]]$ for $l \geqq 2$, we obtain

COROLlary 2.2.6. In the notation of Lemma 2.2.4,

$$
\left[x^{n}, g\right] \equiv[x, g]^{n}[x, g, x]^{(n: 2)} \bmod [G,[G, S],[G, S],[G, S]] .
$$

\section{The upper bound.}

3.1. Preliminary remarks. The contents of this section depend almost entirely on the following two results.

Lemma 3.1.1. (Liebeck [3]). Let $p$ be any prime, $n$ and $s$ arbitrary positive integers and $i$ an integer such that $0 \leqq i<p^{s}$. If $r$ is any positive integer and $f=r p^{s}-(r-1) p^{s-1}$ and if we define $K(n, i)$ by

$$
K(n, i)= \begin{cases}(n: i)-\left(n: i+p^{s}\right)+\ldots+(-1)^{\theta}\left(n: i+\theta p^{s}\right)+\ldots & \text { for } p \text { odd }, \\ (n: i)+\left(n: i+p^{s}\right)+\ldots+\left(n: i+\theta p^{s}\right)+\ldots & \text { for } p=2,\end{cases}
$$

then (i) $p^{r}$ divides $K(n, i)$ if $n \geqq f$, (ii) $p^{r}$ does not divide $K(n, i)$ if $n=f-1$. 
LEMMA 3.1.2. Let $G$ be a group generated by a subset $X=\left\{x_{1}, x_{2}, \ldots\right\}$ of its elements. Then, if for some $g \in G$ we have $\left[g, x_{1}, x_{2}, \ldots, x_{t}\right]=1$ in $G$ for all sets of $t$ elements from $X$, it follows that $g \in \zeta_{t}(G)$.

This result is well-known and in any case very easy to prove.

3.2. Let $B$ be a nilpotent group of class $c$ and $h$ an element of $B$ such that $[B, H]$ is of exponent $p^{r}$ (for some prime $p$ and integer $r$ ). We construct a group $G$ (see Wiegold [1]) which contains (an isomorphic copy of) $B$ and a $p^{s}$ th root for $h(s \geqq 1)$. We then prove that $G$ is nilpotent, and in so doing determine an upper bound for the nilpotency class of $G$ in terms of the variables $c, p, r, s$.

We construct $G$ by forming the wreath product $W$ of $B$ with a cyclic group of order $p^{s}$ generated by the element $\alpha$. As we have seen $(\S 2.2), h_{0} \alpha$ is a $p^{s}$ th root for $h$ in $W$. We put $\dagger$ $A=\operatorname{gp}\left\{h_{0} \alpha\right\}$ and $G=\operatorname{gp}\left\{B^{0}, A\right\}$, where $B^{0}$ is the diagonal of $W$. Thus $G$ contains an isomorphic copy of $B$, namely $B^{0}$, and a $p^{s}$ th root, namely $h_{0} \alpha$, for the element $h$ of $B$ ( $B$ and $B^{0}$ now being identified). The reason we choose $G$ rather than $W$ as the embedding group for $B$ and $h_{0} \alpha$ is that $G$ can be shown to be nilpotent given that $B$ is nilpotent and that $[B, H]$ has prime power exponent, whereas $W$ is certainly not nilpotent if $B$ is not a $p$-group of finite exponent (Baumslag [2]).

We now establish

LEMMA 3.2.1. In the wreath product $W=B \mathrm{wr}\{\alpha\}$, where $\alpha$ generates a $q$-cycle ( $q$ arbitrary) and with $A$ defined as above, the subgroup $\left[B^{0}, A\right]$ is generated by all elements of the form $\left[b, h^{k}\right]_{i}$, where $b$ runs over all elements of $B, k$ is any integer, and the suffix $i$ inicates to which component $B_{i}$ of the direct product $\bar{B}$ the commutator $\left[b, h^{k}\right]_{i}$ belongs.

Proof. It is sufficient for our purposes to show that $\left[B^{0}, A\right]$ is contained in the subgroup $J$ of $W$ generated by all such $\left[b, h^{k}\right]_{i}$ and this only shall we do. The reader will easily satisfy himself that all the generators of $J$ belong to $\left[B^{0}, A\right]$, so that $J=\left[B^{0}, A\right]$.

Now $\left[B^{0}, A\right]$ is generated by all elements of the form $\left[b^{0},\left(h_{0} \alpha\right)^{m}\right]$, where $m$ is an arbitrary integer and $b^{0}=b_{0} b_{1} \ldots b_{q-1}$ is an arbitrary element of the diagonal of $W$. Lemma 2.2.3 shows that for $m=(k-1) q+i \geqq 0$,

$$
\left(h_{0} \alpha\right)^{m}=\alpha^{m} h_{1}^{k} h_{2}^{k} \ldots h_{i}^{k} h_{i+1}^{k-1} \ldots h_{q-1}^{k-1} h_{0}^{k-1},
$$

so that, by (2.1.1),

$$
\begin{aligned}
{\left[b^{0},\left(h_{0} \alpha\right)^{m}\right] } & =\left[b^{0}, h_{1}^{k} \ldots h_{i}^{k} h_{i+1}^{k-1} \ldots h_{q-1}^{k-1} h_{0}^{k-1}\right] \quad \text { (since } b^{0} \text { and } \alpha^{m} \text { commute) } \\
& =\left[b, h^{k}\right]_{1} \ldots\left[b, h^{k}\right]_{i}\left[b, h^{k-1}\right]_{i+1} \ldots\left[b, h^{k-1}\right]_{q-1}\left[b, h^{k-1}\right]_{0} .
\end{aligned}
$$

A similar result follows for $m \leqq 0$, on recalling that conjugation by a power $\alpha^{m}$ of $\alpha$ increases indices by $m$. This completes the proof.

We now remark that, if the set $\{b(1), b(2), \ldots\}$ generates $B$, then $W$ can be generated by the set $K_{i}=\left\{b(1)_{i}, b(2)_{i}, \ldots, \alpha\right\}$, each $i(0 \leqq i<q)$ yielding a different generating set.

† The group $G$ has some interesting properties (cf. Kargapolov, Merzlyakov, Remeslennikov [5]). We mention that it is possible to prove that $G^{\prime} \subseteq(\bar{B})^{\prime}=\bar{B}^{\prime}$, the derived group of the direct product of $p^{3}$ isomorphic copies of $B$. Hence $G$ is soluble of the same length as $B$, and $G^{\prime}$ and $B^{\prime}$ have the same nilpotency class. 
Let us now consider the commutator $\left[\left[b, h^{k}\right]_{i}, w(1), w(2), \ldots, w\left(n_{i}\right)\right]$ of $W$, where $b$ is an arbitrary element of $B, k$ an arbitrary integer, $i$ an arbitrary but fixed integer $(0 \leqq i<q)$ and $w(1), \ldots, w\left(n_{i}\right)$ an arbitrary set of $n_{i}$ generators from $K_{i}$. If $n_{i}$ can be chosen such that the above commutator reduces to the unit element of $W$ for all $b \in B$, all $k$ and all sets of $n_{i}$ generators of $W$ from $K_{i}$ ( $i$ fixed), we shall then be able to deduce from Lemma 3.1.2 that the set of all elements $\left[b, h^{k}\right]_{i}$ ( $i$ fixed) is contained in $\zeta_{n_{i}}(W)$. The same procedure may be applied for all integers $i(0 \leqq i<q)$ and, if $N$ denotes the maximum of all the $n_{i}$, we see that the set of all generators of $J$ (and hence $J$ itself) is contained in $\zeta_{N}(W)$. Since $J \supseteq\left[B^{0}, A\right]$ and $W \supseteq G$, it will then follow that

$$
\left[B^{0}, A, N G\right] \subseteq[J, N W]=E .
$$

But $G$ is generated by $B^{0}$ and $A$ and so $G$ is (see Wiegold [6]) a generalized $(N+1)$ th nilpotent product of two nilpotent groups $B^{0}, A$ and so is itself nilpotent of class at most max $\{N+1$, class of $B^{0}$, class of $\left.A\right\}$.

By symmetry $n_{0}=n_{1}=\ldots=n_{q-1}$, and so, if we determine a value for $n_{0}$ using the generating set $K_{0}$ for $W$, we shall then be able to take $N$ as $n_{0}$. Specifically, we consider commutators of the form $\left[\left[b, h^{k}\right]_{0}, w(1), w(2), \ldots, w\left(n_{0}\right)\right]$ and try to find a value for $n_{0}$ such that the above commutator reduces to the identity of $W$ for all $b \in B$, all integers $k$, and all sets of $n_{0}$ elements from $K_{0}$.

We comment on the general case later, but for the moment content ourselves with the following theorem.

3.3. TheOrem 3.3.1. Let $B$ be a nilpotent group of class $c$, and let $h$ be an element lying in $\gamma_{i}(B)$ and generating the subgroup $H$ of $B$. Let the commutator subgroup $[B, H]$ of $B$ be of exponent $p^{r}$, where $p(\neq 2)$ is a prime and r a positive integer, and let $[B,[B, H],[B, H],[B, H]]=E$. Then $B$ can be embedded together with a $p^{s}$ th root for $h(s \geqq 1)$ in a group $G$ having the same solubility length as $B$, whose derived group $G^{\prime}$ has the same nilpotency class (namely, at most $\dagger$ $\left.\left[\frac{1}{2} c\right]\right)$ as that of $B^{\prime}, G$ itself being nilpotent of class at most $\max \left(c,(r+c-i-1) p^{s}-(r-1) p^{s-1}+1\right)$.

Introduction to proof. As described above, we shall consider commutators of $W$ of the form $\left[\left[b, h^{k}\right]_{0}, w(1), w(2), \ldots, w\left(n_{0}\right)\right]$. We shall say that such a commutator is of length $n_{0}$, and if among the $w(1), w(2), \ldots, w\left(n_{0}\right)$ there occur a total of $\beta$ generators from the subset $K_{0}-\alpha$ of $K_{0}$ we shall call such a commutator a $\beta$-commutator. Thus in a 0 -commutator all the $w(1), \ldots, w\left(n_{0}\right)$ are equal to $\alpha$.

The above commutator can clearly be written in the form

$$
\left[x_{0}, \mu_{1} \alpha, b(1)_{0}, \mu_{2} \alpha, \ldots, \mu_{n} \alpha, b(n)_{0}, \mu_{n+1} \alpha, \ldots\right],
$$

where we have written $x$ for $\left[b, h^{k}\right]$ and $\mu_{1} \alpha$ indicates $\mu_{1}$ successive commutations by $\alpha$. Note that any of $\mu_{1}, \mu_{2}, \ldots$ may well be zero. The $b(1), \ldots, b(n)$ are arbitrary elements from the given generating set for $B$.

We can now begin the proof. From now on $q$ will take the specific value $p^{s}$.

$\dagger[x]$ denotes the greatest integer less than or equal to $x$. 
Proof. Using (2.2.2) we write, on abbreviating $\lambda\left(i, \mu_{j}\right)$ to $\lambda(i, j)$,

It follows that

$$
\left[x_{0}, \mu_{1} \alpha\right]=\prod_{i=0}^{q-1} x_{i}^{\lambda(i, 1)} \text {. }
$$

$$
\left[x_{0}, \mu_{1} \alpha, b(1)_{0}\right]=\left[x^{\lambda(0,1)}, b(1)\right]_{0} .
$$

This result easily extends to

$$
\begin{aligned}
{\left[x_{0}, \mu_{1} \alpha, b(1)_{0}, \ldots, \mu_{n} \alpha, b(n)_{0}\right] } & =\left[\left[\left[\ldots x^{\lambda(0,1)}, b(1)\right]^{\lambda(0,2)}, \ldots, b(n-1)\right]^{\lambda(0, n)}, b(n)\right]_{0} \\
& \in[B, H, n B]_{0} \quad(n=1,2, \ldots) .
\end{aligned}
$$

Let us define, for integers $\rho, \sigma \geqq 0$,

$$
\begin{aligned}
& f(0,0)=0 \\
& f(\rho, \sigma)=\rho p^{s}-(\sigma-1) p^{s-1} \quad \text { otherwise. }
\end{aligned}
$$

(i) If (3.3.2) is a 0-commutator, it takes the form (3.3.3) and if $\mu_{1} \geqq f(r, r)$, Lemma 3.1.1 tells us that all the $\lambda^{\prime}$ 's are multiples of $p^{r}$, so that $\left[x_{0}, \mu_{1} \alpha\right]$ reduces to the unit element of $W$, since $[B, H]$ has exponent $p^{r}$. have

(ii) If (3.3.2) is a 1-commutator, it takes the form $\left[x_{0}, \mu_{1} \alpha, b(1)_{0}, \mu_{2} \alpha\right]$. By (3.3.4), we

and so, by (2.2.2),

$$
\left[x_{0}, \mu_{1} \alpha, b(1)_{0}\right]=\left[x^{\lambda(0,1)}, b(1)\right]_{0}
$$

$$
\left[x_{0}, \mu_{1} \alpha, b(1)_{0}, \mu_{2} \alpha\right]=\prod_{i=0}^{q-1}\left[x^{\lambda(0,1)}, b(1)\right]_{i}^{\lambda(i, 2)} .
$$

We now use Corollary 2.2.6 and the fact that $[B,[B, H],[B, H],[B, H]]=E$ to write

$$
\left[x^{\lambda(0,1)}, b(1)\right]=[x, b(1)]^{\lambda(0,1)}[x, b(1), x]^{v},
$$

where $v=(\lambda(0,1): 2)$. Hence

$$
\left[x^{\lambda(0,1)}, b(1)\right]_{i}^{\lambda(i, 2)}=[x, b(1)]_{i}^{\lambda(0,1) \cdot \lambda(i, 2)}[x, b(1), x]_{i}^{v \cdot \lambda(i, 2)},
$$

since $[x, b(1)]$ and $[x, b(1), x]$ commute $\bmod [B,[B, H],[B, H],[B, H]]$.

Now suppose that $\mu_{1}+\mu_{2} \geqq f(r+1, r)-1$ and that $f\left(r_{1}, r_{1}\right) \leqq \mu_{1}<f\left(r_{1}+1, r_{1}+1\right)$ $\left(r_{1} \geqq 0\right)$. It follows that $\mu_{2} \geqq f\left(r-r_{1}, r-r_{1}\right)$ and so $\lambda(0,1)$ is a multiple of $p^{r_{1}}$ and $\lambda(i, 2)$ is a multiple of $p^{r-r_{1}}$, by Lemma 3.1.1. Since $p \neq 2, v$ is also a multiple of $p^{r_{1}}$, and so

$$
\left[x^{\lambda(0,1)}, b(1)\right]_{i}^{\lambda(i, 2)}=1_{i} \text {, }
$$

since the exponents of both elements on the right hand side of (3.3.8) are then multiples of $p^{r}$.

$\dagger \nu$ is not necessarily a multiple of $p^{r_{1}}$ if $p=2$, so we must employ Corollary 2.2.6 in the form

$$
\left[x^{n}, g\right] \equiv[x, g]^{n} \bmod [B,[B, H],[B, H]] \text {. }
$$

This remark also applies to Theorem 4.1. 
Thus any 1-commutator of length $f(r+1, r)$ reduces to the unit element of $W$.

(iii) If (3.3.2) is a 2-commutator, we begin by noting from (3.3.6) and (3.3.8) that

$$
\left[x_{0}, \mu_{1} \alpha, b(1)_{0}, \mu_{2} \alpha, b(2)_{0}\right]=\left[[x, b(1)]_{0}^{v(1)}[x, b(1), x]_{0}^{v(2)}, b(2)_{0}\right],
$$

where $v(1)=\lambda(0,1) \cdot \lambda(0,2)$ and $v(2)=v \cdot \lambda(0,2)$. By $(2.1 .2)$ and the fact that

$$
[B,[B, H],[B, H],[B, H]]=E,
$$

the right-hand side of (3.3.9) becomes

$$
\left[[x, b(1)]^{v(1)}, b(2)\right]_{0}[x, b(1), x, b(2)]_{0}^{(2)},
$$

and by applying Corollary 2.2 .6 to the first term, since $[x, b(1)] \in[B, H],(3.3 .10)$ becomes

$$
[[x, b(1)], b(2)]_{0}^{v(1)} \cdot[[x, b(1)], b(2),[x, b(1)]]_{0}^{(1): 2} \cdot[x, b(1), x, b(2)]_{0}^{v(2)} .
$$

From all this it follows that

$$
\begin{aligned}
& {\left[x_{0}, \mu_{1} \alpha, b(1)_{0}, \mu_{2} \alpha, b(2)_{0}, \mu_{3} \alpha\right]} \\
& \quad=\prod_{i=0}^{q-1}\left\{[x, b(1), b(2)]_{i}^{v(1)} \cdot[[x, b(1)], b(2),[x, b(1)]]_{i}^{(v(1): 2)} \cdot[x, b(1), x, b(2)]_{i}^{\psi(2)}\right\}^{\alpha(i, 3)},
\end{aligned}
$$

where $\lambda(i, 3)$ is a multiple of $p^{r_{3}}$ if $\mu_{3} \geqq f\left(r_{3}, r_{3}\right)$. The $\lambda(i, 3)$ can be taken inside the product since everything involved in the product commutes.

Now if $\mu_{1}+\mu_{2}+\mu_{3} \geqq f(r+2, r)-2$ and if $f\left(r_{i}, r_{i}\right) \leqq \mu_{i}<f\left(r_{i}+1, r_{i}+1\right)(i=1,2)$, then $\mu_{3} \geqq f\left(r-r_{1}-r_{2} ; r-r_{1}-r_{2}\right)$ owing to the inequality concerning $\mu_{1}+\mu_{2}+\mu_{3}$.

Hence $\lambda(0,1)$, and consequently the $v($ since $p \neq 2)$, are multiples of $p^{r_{1}} . \lambda(0,2)$ is a multiple of $p^{r_{2}}$; consequently $v(1), v(2)$ and $(v(1): 2)$ are multiples of $p^{r_{1}+r_{2}}$. Finally $\lambda(i, 3)$ is a multiple of $p^{r-r_{1}-r_{2}}$ and so all the exponents on the right-hand side of (3.3.12) are multiples of $p^{r}$. That is, every 2-commutator of length at least $f(r+2, r)$ reduces to the unit element of $W$.

(iv) Having shown how the working goes we can proceed by induction on $\beta$. We assume (cf. (3.3.6) together with (3.3.8) and (3.3.12)) that (3.3.2) is a $\beta$-commutator and that it can be written as a product of $\beta+1$ elements, of which the first belongs to $[B, H]_{i}$ and the remaining $\beta$ belong to $[B,[B, H],[B, H]]_{i}$. Further, if $\mu_{j} \geqq f\left(r_{j}, r_{j}\right)(j=1,2, \ldots, \beta+1)$, the powers to which the terms are raised are all muitiples of $p^{r_{1}+r_{2}+\ldots+r_{\beta+1}}$. We then commutate by $b(\beta+1)_{0}$ and then by $\mu_{\beta+2}$ copies of $\alpha$. Repetition of (3.3.9) to (3.3.12) then completes the argument.

(v) Now we assume that $h \in \gamma_{i}(B)$. Hence $[B, H] \subseteq \gamma_{i+1}(B)$ and so, looking at (3.3.4) with $n=c-i$, we see that, if (3.3.2) has more than $c-i-1$ of the generators $b(1)_{0}, b(2)_{0}, \ldots$, it will at once reduce to the unit element of $W$.

If we look at the commutator (3.3.2) and assume its length to be at least

$$
(r+c-i-1) p^{s}-(r-1) p^{s-1}=f(r+c-i-1, r),
$$


we see that (3.3.2) is either at least a $(c-i)$-commutator (i.e. contains at least $c-i$ of the generators $\left.b(1)_{0}, b(2)_{0}, \ldots\right)$ or at most a $(c-i-1)$-commutator containing at least

$$
f(r+c-i-1, r)-(c-i-1)
$$

copies of $\alpha$. In either case we deduce from the above work that such a commutator is the unit element of $W$. Thus we have found a value for $n_{0}=N$ and so, by the discussion preceding Theorem 3.3.1, the theorem is proved.

4. The general case. We again consider commutators of type (3.3.2). Let $h \in \gamma_{i}(B)$ and for the moment assume that $i+1<\frac{1}{3} c$, where $c$ is the class of $B$. Let $j$ be the smallest integer such that $j \geqq \frac{1}{3} c$ and let $k=j-i-1$. Then $k>0$. Consider a commutator of type (3.3.2) of length $k . f(r, r)$, where, as before, $p^{r}$ is the exponent of $[B, H]$. Such a commutator is at least a $k$-commutator or at most a $(k-1)$-commutator containing at least $k \cdot f(r, r)-(k-1)$ copies of $\alpha$. In this case at least one of the $\mu$ 's (there are at most $k$ of them) is greater than or equal to $f(r, r)$, and so (3.3.2) will reduce to the unit element. If (3.3.2) is of the former type, that is at least a $k$-commutator, we consider the "subcommutator" of (3.3.2) as far as the term $b(k)_{0}$, that is (3.3.4) with $n=k$. This subcommutator then belongs to

$$
[B, H, k B]_{0} \subseteq \gamma_{i+1+k}\left(B_{0}\right)=\gamma_{j}\left(B_{0}\right) .
$$

Call this subcommutator $X_{0}$. Hence $X_{0} \in[B, H, k B]_{0} \subseteq[B, H]_{0}$ and so has order $p^{r}$ at most. Now $\left[B, \gamma_{j}(B), \gamma_{j}(B), \gamma_{j}(B)\right]=E$, since $j \geqq \frac{1}{3} c$ and $\gamma_{j}(B)=\left[\gamma_{j-1}(B), B\right]$ so that, by Corollary 2.2.6 with $G=B$ and $S=\gamma_{j-1}(B)$, we have, for any $y \in \gamma_{j}(B)$ and $b \in B$,

$$
\left[y^{n}, b\right]=[y, b]^{n}[y, b, y]^{(n: 2)} \text {. }
$$

We now proceed in exactly the same way as in $\S 3.3$, and we see that, since $X_{0} \in \gamma_{j}\left(B_{0}\right)$, the commutator $\left[X_{0}, w(1), w(2), \ldots, w\left(n_{x}\right)\right]=1$ in $W\left(\right.$ where $\left.w(1), w(2), \ldots, w\left(n_{x}\right) \in K_{0}\right)$, provided that $n_{x} \geqq f(r+c-j, r) \quad$ (cf. Theorem 3.3.1 (iv)). But $X_{0}$ was itself of length at most $k . f(r, r)$ and so any commutator of type (3.3.2) of length at least $(j-i-1) f(r, r)+f(r+c-j, r)=Z$, say, is the unit element of $W$.

Thus the set of all $\left[b, h^{k}\right]_{0} \in \zeta_{\mathrm{Z}}(W)$ and so $\left[B^{0}, A, Z G\right]=E$ in $G$. Hence $G$ is nilpotent of class at most $\max (c, Z+1)(p \neq 2)$. If $i+1 \geqq \frac{1}{3} c$, then $[B,[B, H],[B, H],[B, H]]=E$ and so Theorem 3.3.1 applies. Precisely, we have

THEOREM 4.1. Let $B$ be nilpotent of class $c$ and let $h \in \gamma_{i}(B), H=\mathrm{gp}\{h\} . \quad$ Let $[B, H]$ have exponent $p^{r}(p \neq 2)$. Then $B$ can be embedded with a $p^{s}$ th root for $h(s \geqq 1)$ in a group $G$ having the same solubility length as $B, G^{\prime}$ and $B^{\prime}$ having the same nilpotency class, $G$ itself being nilpotent of class at most

$$
\left\{\begin{array}{l}
\max \left\{c,(j-i-1)\left(r p^{s}-(r-1) p^{s-1}\right)+(r+c-j) p^{s}-(r-1) p^{s-1}+1\right\}, \quad \text { if } i+1<\frac{1}{3} c, \\
\max \left\{c,(r+c-i-1) p^{s}-(r-1) p^{s-1}+1\right\}, \quad \text { if } i+1 \geqq \frac{1}{3} c,
\end{array}\right.
$$

where $j$ is the integer such that $\frac{1}{3} c \leqq j<\frac{1}{3} c+1$.

For $i=1$, the first bound here is approximately one third that obtained by Wiegold [1], which was (on correcting a minor error) $(c-1)\left(r p^{s}-(r-1) p^{s-1}\right)+1$. 
5. Lower bounds. If $B$ is any nilpotent group of class $c$ contained in the nilpotent group $G$, then the class of $G$ is not less than $c$. As stated in the introduction, Wiegold has constructed, for each odd integer $r \geqq 3$, a class 2 group $B_{r}$ containing an element $h$ and for which $[B, H]$ has exponent $p^{r}$, such that no nilpotent group $G_{r}$ containing $B_{r}$ and a $p$ th root for $h$ has class less than $\frac{1}{2}(r+5)$. If we restrict attention to those groups $G_{r}$ having the same solubility length as $B_{r}$, we can prove slightly more, namely, that the class of $G_{r}$ also depends on $p$.

We require the following lemmas.

Lемма 5.1. If $x, y$ are arbitrary elements of the metabelian group $X$ and $n$ is an arbitrary positive integer, the following equality holds:

$$
\left[x, y^{n}\right]=[x, y]^{n}[x, 2 y]^{(n: 2)} \ldots[x, n y] .
$$

The proof follows easily by induction on $n$.

Lemma 5.3. If $x_{1}, x_{2}, \ldots, x_{n}$ are arbitrary elements of the derived group of the metabelian group $X$ and if $y$ is an arbitrary element of $X$, we have, for $\varepsilon_{i}= \pm 1$,

$$
\left[x_{1}^{\varepsilon_{1}} x_{2}^{\varepsilon_{2}} \ldots x_{n}^{\varepsilon_{n}}, y\right]=\left[x_{1}, y\right]^{e_{1}}\left[x_{2}, y\right]^{\varepsilon_{2}} \ldots\left[x_{n}, y\right]^{\varepsilon_{n}} .
$$

The proof is straightforward.

The group $B_{r}$ constructed by Wiegold was given by

$$
B_{r}=\mathrm{gp}\left\{b, h ; b^{p^{r}}=h^{p^{r}}=[h, b, b]=[h, b, h]=1\right\} .
$$

It is clear that $B_{r}$ has class 2 and is consequently metabelian. Without loss of generality, we take $G_{r}$ to be a metabelian nilpotent group generated by $B_{r}$ and an element $a$ such that $a^{p}=h$. We shall prove that $G_{r}$ is nilpotent of class greater than $p$. In $B_{r},[b, h]^{p^{r}}=\left[b^{p^{r}}, h\right]=1$, whilst $[b, h]^{p^{r-1}}=\left[b^{p^{r-1}}, h\right] \neq 1$. Putting $b=b^{p^{r-1}}$, we have $\left[b^{p}, h\right]=1,[b, h] \neq 1$ in $B_{r}$. Further, $G_{r}$ is generated by elements of $p$-power order and so is a $p$-group. Hence the elements $[b, a],[b, a]$ have $p$-power order. Since $G_{r}$ is metabelian, $\left[B_{r}, A\right]$ is abelian $(A=\mathrm{gp}\{a\})$ and since $B_{r}, A$ generate $G_{r},\left[B_{r}, A\right] \triangleleft G$. Now in $G_{r}$,

$$
1 \neq[b, h]=\left[b, a^{p}\right]=[\bar{b}, a]^{p}[b, 2 a]^{(p: 2)} \ldots[\bar{b}, p a],
$$

(by 5.2), and since the order of $[b, \overline{i+1} a]$ divides that of $[b, i a](i=1,2, \ldots)$ (this follows since $[b, \overline{i+1} a]=[b, i a]^{-1} a^{-1}[b, i a] a$ and $\left[B_{r}, A\right]$ is abelian normal in $G_{r}$ ), we deduce that, if $[\bar{b}, a]$ has order $p$, then $[b, p a] \neq 1$ in $G_{r}$. That is, the class of $G$ is greater than $p$.

If, on the other hand, $[\bar{b}, a]$ has order $p^{v}$, say, where $v>1$, we write

$$
1=\left[a, b^{p}\right]=[a, b]^{p}[a, 25]^{(p: 2)} \ldots[a, p b] .
$$

Using the argument above, we deduce that, if $[a, i b]$ has order $p^{v_{1}}$, then $[a, \overline{i+1} b]$ has order $p^{v_{i+1}}$, where $v_{i} \geqq v_{i+1}$. That is, $v_{1} \geqq v_{2} \geqq \ldots \geqq v_{p}$.

We now write the above equation in the form

$$
[a, p b]=[a, b]^{-p}[a, 2 b]^{-(p: 2)} \ldots[a, \overline{p-1} b]^{-(p: p-1)},
$$

and commutate on each side by a further $\bar{b}$, giving (by 5.4)

$$
[a, \overline{p+1} b]=[a, 2 \bar{b}]^{-p}[a, 3 \bar{b}]^{(-p: 2)} \ldots[a, p \bar{b}]^{-(p: p-1)} .
$$


We now substitute for the final term using (5.5), noting that all terms are permutable since $G$ is metabelian, to obtain

$$
[a, \overline{p+1} b]=[a, \bar{b}]^{t_{1}}[a, 25]^{t_{2}} \ldots[a, \overline{p-1} b]^{t_{p}-1},
$$

where $t_{1}$ is a multiple of $p^{2}$ but not of $p^{3}$ and $t_{2}, t_{3}, \ldots, t_{p-1}$ are multiples of $p$ and not of $p^{2}$. Continuing this process $j$ times (where $j$ is an arbitrary integer such that $1 \leqq j \leqq p-1$ ) we easily obtain, again using induction,

$$
[a, \overline{p+j} b]=[a, b]^{t_{1}}[a, 2 b]^{t_{2}} \ldots[a, \overline{p-1} b]^{t_{p-1}},
$$

where now $t_{1}, t_{2}, \ldots, t_{j}$ are multiples of $p^{2}$ but not of $p^{3}$ and $t_{j+1}, \ldots, t_{p-1}$ are multiples of $p$ but not of $p^{2}$.

Now let $v_{i}$ be such that $v_{1}=v_{2}=\ldots=v_{i}>v_{i+1} \geqq v_{i+2} \geqq \ldots \geqq v_{p-1}$ and take (5.8) with $j=i-1$. We obtain

$$
[a, \overline{p+i-1} b]=[a, b]^{t_{1}}[a, 2 \bar{b}]^{t_{2}} \ldots[a, \overline{p-1} b]^{t_{p-1}},
$$

where $t_{1}, t_{2}, \ldots, t_{i-1}$ are multiples of $p^{2}$ but not of $p^{3}$ and $t_{i}, t_{i+1}, \ldots, t_{p-1}$ are multiples of $p$ but not of $p^{2}$. Raising each side of (5.9) to the power $p^{v_{1}-2}$ and remembering that $v_{1} \geqq 2$, we have, putting $v_{1}=v$,

$$
[a, \overline{p+i-1} b]^{p^{\nu-2}}=[a, b]^{w_{1}}[a, 2 \bar{b}]^{w_{2}} \ldots[a, \overline{p-1} b]^{w_{p-1}},
$$

where $w_{1}, w_{2}, \ldots, w_{i-1}$ are multiples of $p^{\nu}$ but not of $p^{\nu+1}$ and $w_{i}, w_{i+1}, \ldots, w_{p-1}$ are multiples of $p^{v-1}$ but not of $p^{v}$. Equation (5.10) then reduces to

$$
[a, \overline{p+i-1} \bar{b}]^{p^{\nu-2}}=[a, i \bar{b}]^{p^{\nu-1}}
$$

all other terms on the right hand side of (5.10) reducing to the unit element of $G$. We deduce that $[a, \overline{p+i-1} b]^{p^{\nu-2}} \neq 1$ in $G$, and consequently $[a, \overline{p+i-1} b]$ and, even more so, $[a, p \bar{b}] \neq 1$ in $G$. That is, the class of $G$ is greater than $p$.

\section{REFERENCES}

1. J. Wiegold, Adjunction of elements to nilpotent groups, J. London. Math. Soc. 38 (1963), 17-26.

2. G. Baumslag, Wreath products and p-groups, Proc. Cambridge Philos. Soc. 55 (1959), 224-231. 443-451.

3. H. Liebeck, Concerning nilpotent wreath products, Proc. Cambridge Philos. Soc. 58 (1962),

4. O. N. Golovin, Nilpotent products of groups, Mat. Sbornik 27 (69) (1950), 427-454. Amer. Math. Soc. Transl. vol. 2, Ser. 2.2 (1956), 89-115.

5. M. I. Kargapolov, Ju. I. Merzlyakov, V. N. Remeslennikov, Completion of groups, Dokl. Akad. Nauk. S.S.S.R. 134 (1960), 518-520.

6. J. Wiegold, Nilpotent products of groups with amalgamations, Pub. Math. Debrecen 6 (1959) 131-168.

\section{UNIVERSITY COLLEGE CARDIFF}

